\title{
Modeling and Simulation of Current Distribution of Wire Antenna Based on MOM
}

\author{
Qiaoqiao Li \\ School of Electrical and Electronic Engineering, North China Electric Power University, Beijing \\ 102200 010, China \\ qiaoqiaocindy@126.com
}

Keywords: MOM; Antenna simulation; MATLAB

\begin{abstract}
This paper introduces a method to solve the current distribution of half-wave dipole by using MOM. In the three-dimensional coordinate system, the z-coordinate is segmented uniformly. The pulse function is selected as the basis function and the impulse function as the matching function. The Pocklington equation is solved by MATLAB, and the current convergence value is obtained. The results shows MOM can be used to analyzes thin dipole antenna effectively.
\end{abstract}

\section{Introduction}

With the rapid development of high-speed computer, high-precision approximation method have substituted the previous time-consuming and laborious analytical method to solve more problems faster. The basic idea of the scholars' research is to convert the functional equation into a matrix equation, and then solve the matrix equation by MATLAB. MOM is a numerical method for strictly calculating the electromagnetic radiation and scattering in the open field. It integrates the continuous equation into an algebraic equation and is suitable for solving the micro and integral equations. Line antennas are the simplest three-dimensional problem in the field of electromagnetic field calculations, but their practical applications are extremely extensive. Currently, on the system of electromagnetic compatibility procedures, there are no open source code. It is difficult to analyze the software and make secondary development. From this situation, this paper establishes the mathematic model of the feeder antenna have developed the corresponding program by MOM to provide the basis and reference for the future research.

\section{The Basic Idea}

First, the thin wires are discretized, generally with the maximum segment length as one-twentieth wavelength. Secondary, we should choose the basis function and separate the Pocklington integral equation into a linear algebraic system. Thirdly, the current on the line segments of the linear algebraic equations is obtained. Finally, the electric dipole formula and the superposition theorem are used to calculate the electric field intensity and other indexes of any point in the space.

\section{Model Construction}

In this section, we will build a math model of the line current to calculate its current distribution. To be specific, we are going to discuss the emission characteristic of line antenna here. We also want to note that, for the emission and scattering problems, only the value of some parameters are different, the analysis method is the same.

\section{Fundamental and Preparation}

Fundamental of Wire Antenna. According to related theory of engineering electromagnetic field, the electric field intensity formula of the fine line current and the current Pocklington integral equation are as follows [2] 


$$
\mathrm{Z}_{\text {wire }}=\mathrm{e}_{1} \mathrm{E}_{\mathrm{e}}-\mathrm{j} \frac{\mathrm{l}}{4 \pi \mathrm{k}} \mathrm{e}_{\mathrm{l}} \int_{1} \mathrm{I}\left(\mathrm{l}^{\prime}\right)\left(\mathrm{k}^{2} \mathrm{e}_{\mathrm{l}^{\prime}}-\nabla \frac{\partial}{\partial \mathrm{l}^{\prime}}\right) \mathrm{G}\left(\mathrm{r}, \mathrm{r}^{\prime}\right) \mathrm{dl} \mathrm{l}^{\prime}
$$

$\mathrm{G}\left(\mathrm{r}, \mathrm{r}^{\prime}\right)$ is the Green function.

$$
\mathrm{G}\left(\mathrm{r}, \mathrm{r}^{\prime}\right)=\frac{\mathrm{e}^{-\mathrm{jk}\left|\mathrm{r}-\mathrm{r}^{\prime}\right|}}{4 \pi\left|\mathrm{r}-\mathrm{r}^{\prime}\right|}
$$

For the emission problem, the external electric field strength is 0 . The voltage of the feed port is always already known, and the current distribution along the thin wire satisfies the following equation.

$$
\frac{\mathrm{U}_{s}}{\mathrm{~h}}=-\mathrm{j} \frac{\eta}{4 \pi \mathrm{k}} \mathrm{e}_{1} \cdot \int_{\mathrm{L}} \mathrm{I}\left(\mathrm{l}^{\prime}\right)\left(\mathrm{k}^{2} \mathrm{e}_{1},-\nabla \frac{\partial}{\partial 1^{\prime}}\right) \mathrm{G}\left(\mathrm{r}, \mathrm{r}^{\prime}\right) \mathrm{dl}^{\prime}
$$

It is the distance between the two ends of the thin wire antenna supply. (In the later discretization, for a discrete element after discrete, $\mathrm{U}_{\mathrm{s}}$ is the voltage at both ends of the feed point, and $\mathrm{h}$ is the length of the minute). Once the current distribution on the thin wire is obtained, the electric field strength at any point in the space can be calculated.

$$
\mathrm{E}(\mathrm{r})=-\mathrm{j} \frac{\eta}{4 \pi \mathrm{k}} \mathrm{e}_{1} \cdot \int_{\mathrm{L}} \mathrm{I}\left(\mathrm{l}^{\prime}\right)\left(\mathrm{k}^{2} \mathrm{e}_{1},-\nabla \frac{\partial}{\partial \mathrm{l}^{\prime}}\right) \mathrm{G}\left(\mathrm{r}, \mathrm{r}^{\prime}\right) \mathrm{dl}^{\prime}
$$

Base Function Selection. Considering the simplicity of the algorithm, the basis function is chosen as the pulse function.

$$
\mathrm{I}=\sum_{\mathrm{n}=0}^{\mathrm{N}} \alpha_{\mathrm{n}} \mathrm{I}_{\mathrm{n}}
$$

(5) $\quad I_{n}= \begin{cases}1, & \text { On the } n-\text { th segment of the antenna } \\ 0, & \text { On the other segments of the antenna }\end{cases}$

The Test Function Selection. We select the test function as the impulse function, which is also called the point matching method. And then, we can construct the matrix elements.

\section{Model Assumption}

The conductor as $\mathrm{N}$-series together, can be imagined as an N-port network. Once the impedance matrix is obtained, the admittance matrix can be found, which we can use to find the port current, so as to find the conductor current distribution.

Since it is a thin wire, it is assumed that the current flows only in the direction of the wire shaft. The surface current is ignored because the radius is much smaller than the total length

The right side of the equation calculates the electric field strength of the wire surface.

\section{Discretization of the Equations}

Firstly, we establish the coordinate system, with the line current on the $\mathrm{Z}$ axis and the coordinate origin is the end of the line antenna, and then the wave Clinton equation can be written as follows.

$$
\mathrm{E}_{z}=-\frac{1}{\mathrm{j} \omega z}\left(\frac{\partial^{2}}{\partial z^{2}}+\mathrm{k}^{2}\right) \int_{l_{z^{\prime}}} \frac{\mathrm{I}_{z^{\prime}} \mathrm{e}^{-\mathrm{jkR}}}{4 \pi \mathrm{R}} \mathrm{dz} z^{\prime}
$$

The base function is brought into the equation and discretized.

$$
\begin{aligned}
& \mathrm{E}_{z \mathrm{~m}}=-\frac{1}{\mathrm{j} \omega z}\left(\frac{\partial^{2}}{\partial z^{2}}+\mathrm{k}^{2}\right) \sum_{\mathrm{i}=1}^{\mathrm{N}} \alpha_{\mathrm{n}} \int_{\Delta 1_{\mathrm{n}}} \frac{\mathrm{e}^{-\mathrm{jkR}}}{4 \pi \mathrm{R}} \mathrm{d} \\
& (7) \mathrm{m}=0,1,2 \ldots \ldots \mathrm{M}
\end{aligned}
$$

Therefore, the generalized voltage on the commutation segment can be expressed as follows.

$$
\mathrm{V}_{\mathrm{m}}=\Delta \mathrm{l}_{\mathrm{m}} \mathrm{E}_{\mathrm{zm}}=\sum_{\mathrm{n}=1}^{\mathrm{N}} \alpha_{\mathrm{n}} \frac{\Delta \mathrm{l}_{\mathrm{m}}}{\mathrm{j \omega z}}\left(\frac{\partial^{2}}{\partial z^{2}}+\mathrm{k}^{2}\right) \int_{\Delta 1_{\mathrm{n}}} \frac{\mathrm{e}^{-\mathrm{jkR}}}{4 \mathrm{mR}} \mathrm{d} z^{\prime}
$$


As mentioned above, this line antenna is treated as an N-port network, and the voltage applied to each port is approximately to $V_{j}$.

We define the matrix as $[\mathrm{I}]=\left[\begin{array}{c}\alpha_{1} \\ \alpha_{2} \\ \vdots \\ \alpha_{\mathrm{N}}\end{array}\right], \quad[\mathrm{V}]=\left[\begin{array}{c}\Delta \mathrm{l}_{1} \cdot \mathrm{E}_{\mathrm{z} 1} \\ \Delta \mathrm{l}_{2} \cdot \mathrm{E}_{\mathrm{zz}} \\ \vdots \\ \Delta \mathrm{l}_{\mathrm{M}} \cdot \mathrm{E}_{\mathrm{zM}}\end{array}\right]$

$[\mathrm{V}]=[\mathrm{Z}][\mathrm{I}]$

Calculating $\mathrm{Z}$, we can derive the impedance element by studying the intensity of the electric field on the surface of a certain section of the axis.

Finite Difference Method.

$$
\psi(\mathrm{n}, \mathrm{m})=\frac{1}{4 \pi \Delta \mathrm{l}_{\mathrm{n}}} \int_{\Delta \mathrm{l}_{\mathrm{n}}} \frac{\mathrm{e}^{-\mathrm{jkR} \mathrm{m}}}{4 \pi \mathrm{R}_{\mathrm{m}}} \mathrm{dz}
$$

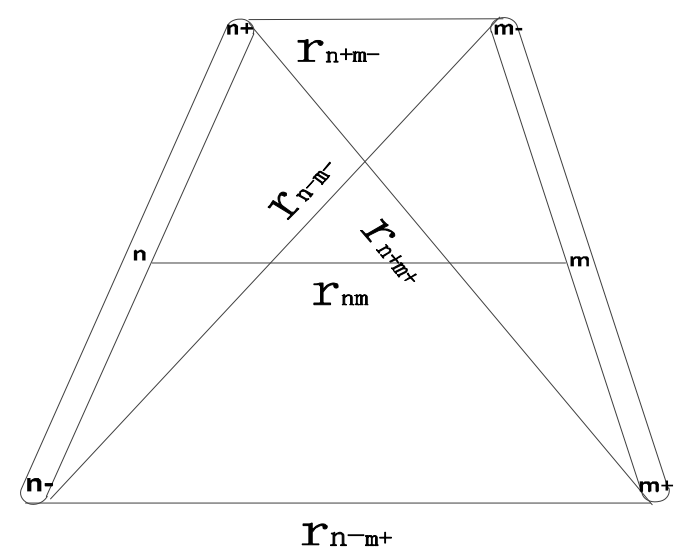

Figure 1. Conductor segmentation diagram

In this formula, $\mathbf{R}_{\mathbf{m}}$ is the distance from the previous point on $\Delta \mathbf{l}_{\mathbf{n}}$ to the $\mathrm{m}$ point. Sign + and added to $\mathrm{m}$ and $\mathrm{n}$ at the appropriate time.

$$
\begin{aligned}
& \mathrm{Z}_{\mathrm{nm}}= \\
& \mathrm{j} \omega \mu \Delta \mathrm{l}_{\mathrm{n}} . \\
& \Delta \mathrm{l}_{\mathrm{m}} \cdot \\
& \psi(\mathrm{n}, \mathrm{m})+ \\
& \frac{1}{\mathrm{j} \omega \mathrm{g}}\left[\mathrm{i}^{+}, \mathrm{n}^{+}\right)- \\
& \psi\left(\mathrm{n}^{-}, \mathrm{m}^{+}\right)- \\
& \psi\left(\mathrm{n}^{+}, \mathrm{m}^{-}\right)+ \\
& \left.\psi\left(\mathrm{n}^{-}, \mathrm{m}^{-}\right)\right]
\end{aligned}
$$

Taylor Unfolds. We use Taylor unfolds to expand the exponent into a McLaughlin series and get the approximate expression.

$$
\Psi(\mathrm{n}, \mathrm{m})=\frac{1}{\Delta \mathrm{I}_{\mathrm{n}}} \int_{\mathrm{n}^{-}}^{\mathrm{n}^{+}} \frac{\mathrm{e}^{-\mathrm{jk} \mathrm{R}_{\mathrm{m}}}}{4 \pi \mathrm{R}_{\mathrm{m}}} \mathrm{dz} \mathrm{z}^{\prime}
$$

(

In the formula, 


$$
\mathrm{R}_{\mathrm{m}}=\left\{\begin{array}{r}
\sqrt{\rho_{\mathrm{m}}^{2}+\left(\mathrm{z}-\mathrm{z}_{\mathrm{m}}\right)^{2}}, \mathrm{~m} \neq \mathrm{n} \\
\sqrt{\rho_{\mathrm{m}}^{2}+\mathrm{z}^{2}}, \mathrm{~m}=\mathrm{n}
\end{array}\right.
$$

(

$\rho_{\mathrm{m}}$ is the radius of the line.

$$
\psi=\frac{1}{4 \pi \Delta l_{\mathrm{n}}} \int_{\mathrm{n}^{-}}^{\mathrm{n}^{+}}\left(\frac{1}{\mathrm{R}_{\mathrm{m}}}-\mathrm{jk}-\frac{\mathrm{k}^{2}}{2} \mathrm{R}_{\mathrm{m}}+\cdots\right) \mathrm{dz}
$$

(

The second term has nothing to do with $\mathrm{R}_{\mathrm{m}}$.

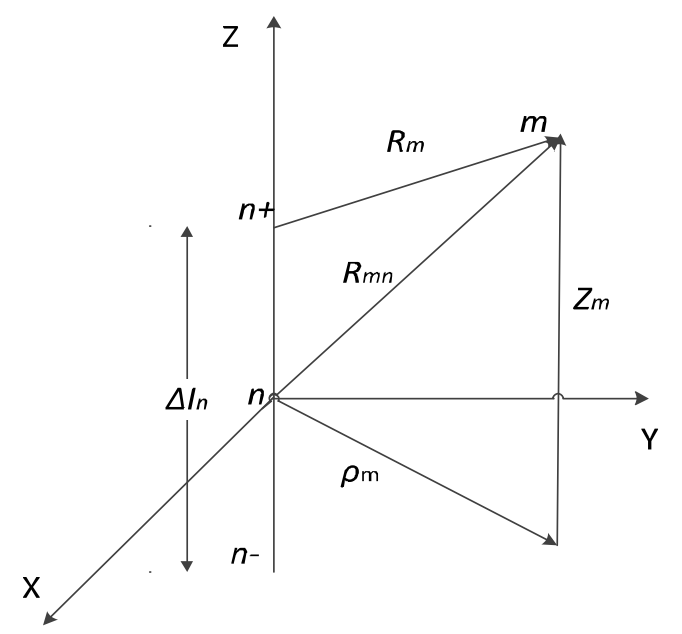

Figure 2. The three - dimensional coordinate diagram of the antenna

When $\mathrm{m}=\mathrm{n}$, by the reference, the accuracy given by the first and second terms is satisfactory.

$$
\Psi(\mathrm{n}, \mathrm{m}) \approx \frac{1}{2 \pi \Delta 1_{\mathrm{n}}} \ln \left(\frac{\Delta \mathrm{l}_{\mathrm{n}}}{\rho_{\mathrm{m}}}\right)-\frac{\mathrm{j} \mathrm{k}}{4 \pi}
$$

When $\mathrm{m} \neq \mathrm{n}$, the rough approximation is to use $\mathrm{R}_{\mathrm{m}}$ as a constant in the integral form.

\section{(16)}

$$
\psi(\mathrm{n}, \mathrm{m}) \approx \frac{\mathrm{e}^{-\mathrm{jkR} \mathrm{n}_{\mathrm{nm}}}}{4 \pi \mathrm{R}_{\mathrm{nm}}}
$$

$\mathrm{R}_{\mathrm{nm}}$ is the distance from $\mathrm{n}$ to $\mathrm{m}$. According to the study of the reference, in the calculation of this example, the approximation error can be ignored.

\section{Model Solving}

The Main Idea of the Program. The solution idea is to examine the impedance $Z_{n m}$ associated with the $n$-th stage current and the m-th field, which must calculate $\psi(\mathrm{n}, \mathrm{m}), \psi\left(\mathrm{n}^{+}, \mathrm{m}^{+}\right), \psi\left(\mathrm{n}^{-}, \mathrm{m}\right), \psi\left(\mathrm{n}^{+}, \mathrm{m}^{-}\right)$and $\psi\left(\mathrm{n}^{-}, \mathrm{m}^{-}\right)$first. There are two cases of investigation of $\psi(n, m)$. When $n=m$, we can expend it by Taylor Expansion Formula, in addition, $\mathrm{R}_{\mathrm{m}}$ is approximated used as a constant solution. 


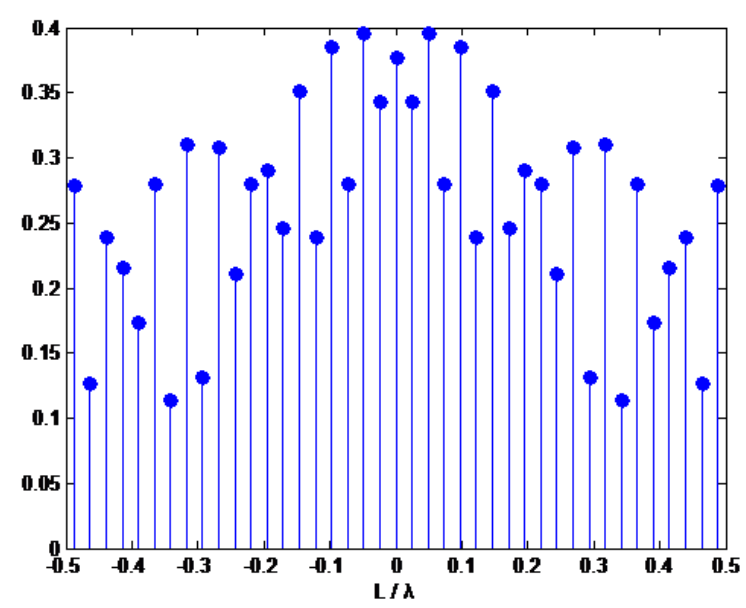

Figure 3. Half - wave antenna current distribution

(The vertical axis is the normalized current)

Discussion. After several experiments, the convergence of the algorithm is excellent when the number of segments $\mathrm{N}=65$, But we find that this algorithm is very error-prone and very complex, because for different cases of $Z_{n m}$, we must separately discuss the different conditions of the five $\Psi$ function.

\section{Program Optimization}

The Main Idea. We do not first press classification to $Z_{n m}$, but to discuss the $\Psi$ function with every different $\mathrm{n}$ and $\mathrm{m}$.

\section{Consequence.}

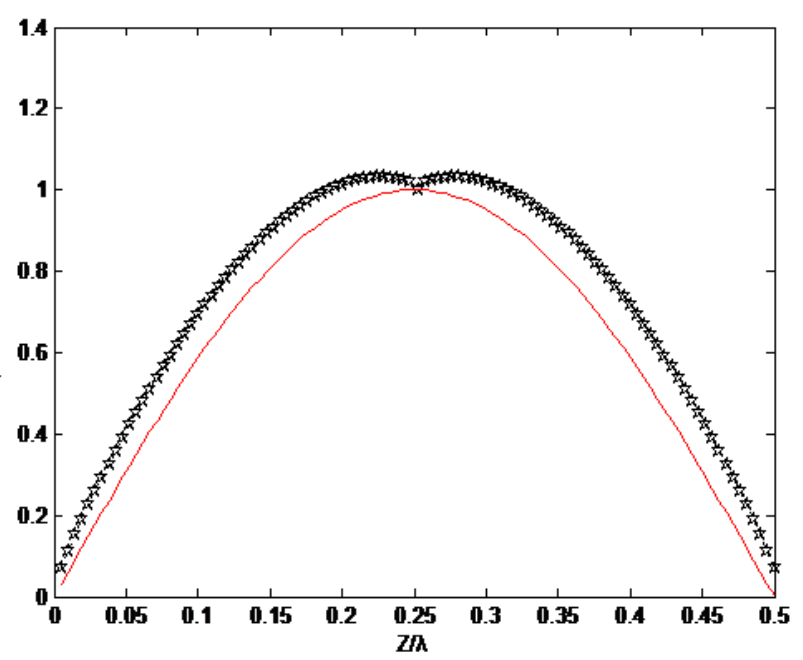

Figure 4. Improved half - wave antenna current distribution

(The vertical axis is the normalized current)

Discussion. As the algorithm is simplified, the total number of rows is significantly reduced, and the error is also significantly reduced. Experiments show that the higher the $\mathrm{N}$, the higher the accuracy of the simulation, and there is no upper limit of convergence, but the lower limit of 105 . 
The current distribution at different wavelengths and line lengths is calculated and found to be consistent with the existing analytical solutions.
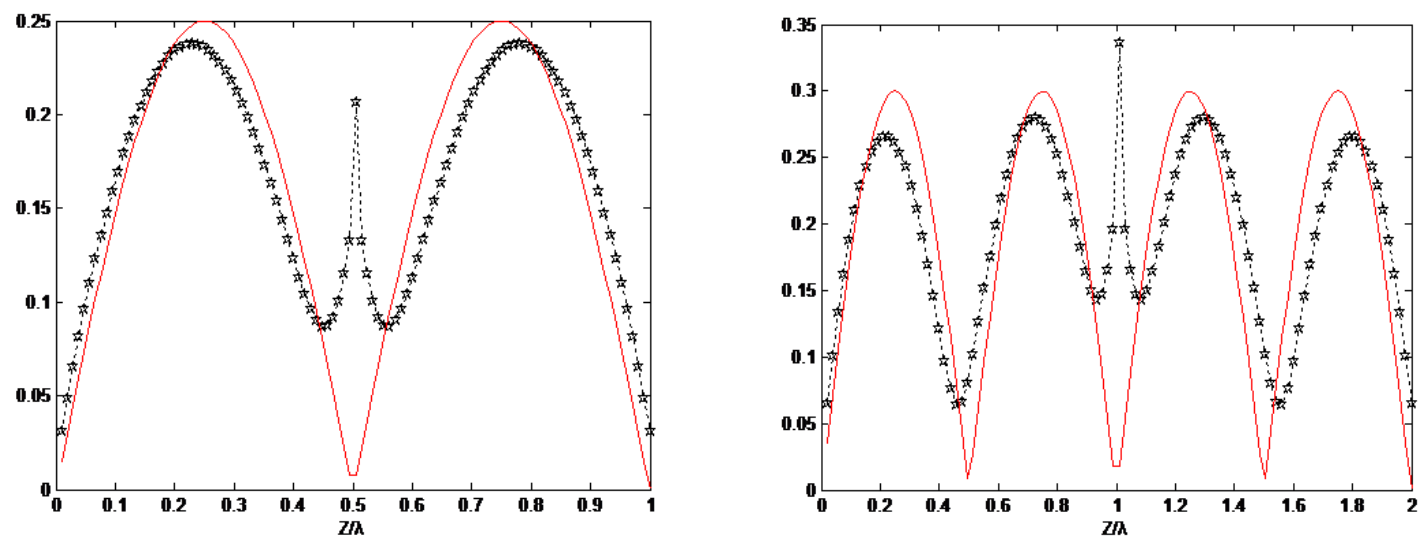

Figure 5. Simulation results of antenna current distribution at different wavelengths

\section{Conclusion and Discussion}

The ratio of and 1 will have an effect on the simulation results, for the reason that, when a is large, the model should be equivalent to a cylinder. Then there is a problem with the approximation of the surface current and other similar assumption. Simultaneously, the current distribution on the axis of the assumption is also a problem either.

Theoretically, the larger the $\mathrm{N}$, the smaller the length $\mathrm{d}$ of each segment, the smaller the approximation error of the $\Psi$ function, so that the simulation error of the whole current distribution is smaller. The improvement process validates the existing theoretical research.

\section{References}

[1] R.F. Harrington: Calculating the Moment Method of Electromagnetic Fields (Beijing: National Defense Industry Press, 1981: $6 \sim 13$ ).

[2] G.Z Ni, X.Y Qian, Electromagnetic Field Numerical Computation (Higher Education Press .1995).

[3] Y.H Lu, Numerical Methods for Calculating Electromagnetics (Beijing: Tsinghua University Press). 